\title{
Flexibilité de la perspective temporelle et les systèmes temporels français et serbe
}

\author{
Veran Stanojević \\ Faculté de Philologie, Université de Belgrade*
}

\begin{abstract}
Nous nous intéressons dans ce travail ${ }^{1}$ au rôle de la position de la perspective temporelle dans l'interprétation des temps verbaux du serbe par comparaison au français. En nous appuyant sur l'approche de Co Vet selon laquelle, en français, la perspective temporelle, située soit au moment de la parole soit à un moment du passé, ne change pas de position dans différents emplois temporels des formes verbales fléchies, nous constatons qu'en serbe, par contre, pour rendre compte de différents emplois des temps verbaux, il est nécessaire de postuler la flexibilité de la perspective temporelle. Ceci explique non seulement certains emplois dits relatifs des temps verbaux en serbe, mais en plus donne des prédictions quant au choix des équivalents français des formes verbales constituant le premier sous-système temporel en serbe.
\end{abstract}

Mots-clés : temps verbaux, aspect, perspective temporelle, le serbe, le français.

\section{Introduction}

La perspective temporelle $(\mathrm{R})$, dont l'ancêtre est le point de référence de Reichenbach (Reichenbach 1947), a été introduite, entre autres, pour éviter de parler du rapport direct entre le procès $(E)$ et le moment de la parole $(S)$ dans les descriptions des temps verbaux en langue naturelle. En effet, le sens temporel de certains temps verbaux du français ne peut pas être défini comme rapport direct entre $\mathrm{E}$ et $\mathrm{S}$, comme c'est le cas, par exemple, du plus-que-parfait que Reichenbach définit E-R-S. Qui plus est, pour certains autres temps le rapport de E à S ne semble pas du tout pertinent. Il s'agit notamment du conditionnel présent en emploi temporel, avec lequel, en fonction du contexte, E peut établir n'importe quel rapport chronologique avec $\mathrm{S}$ : simultanéité $(\mathrm{E}, \mathrm{S})$, antériorité $(\mathrm{E}-\mathrm{S})$ ou postériorité $(\mathrm{S}-\mathrm{E})$ :

\footnotetext{
*veranva@gmail.com

1 Cette recherche contribue au projet scientifique $\mathrm{N}^{\circ} 178002$ (Jezici $i$ kulture $u$ vremenu $i$ prostoru) financé par le Ministère de la Science et du Développement technologique de Serbie.
} 
1) Il a dit (R) qu'il viendrait (E) hier. (R-E-S)

2) Il a dit (R) qu'il viendrait (E) maintenant. (R-E,S)

3) Il a dit (R) qu'il viendrait (E) demain. (R-S-E)

$\mathrm{Au}$ vu de ces exemples, il s'avère que, pour le conditionnel, seuls comptent les rapports entre $\mathrm{R}$ et $\mathrm{S}, \mathrm{R}$ étant dans le passé (R-S), et entre E et R, E étant postérieur à R (R-E). Il s'agit donc de deux invariants sémantiques qui font partie du sémantisme du conditionnel temporel du français. La variabilité de la position de $\mathrm{E}$ par rapport à $\mathrm{S}$ étant fonction du contexte, cette relation temporelle ne figure pas dans le sémantisme du conditionnel.

Déjà dans la théorie classique de Reichenbach, la position de $\mathrm{R}$ par rapport à $\mathrm{S}$ est fixe (à $\mathrm{S}$, avant $\mathrm{S}$, ou après $\mathrm{S}$ ) indépendamment de l'emploi temporel des temps verbaux. Co Vet a montré que la position de $\mathrm{R}$ par rapport à $\mathrm{S}$ est fonction de la morphologie flexionnelle (Vet 1980, 2008). Ainsi, les désinences du présent impliquent que $\mathrm{R}$ est dans $\mathrm{S}$, alors que les désinences de l'imparfait font déplacer $\mathrm{R}$ dans le passé (R-S). En français, pour ce qui est de la position de la perspective temporelle, seules comptent donc les relations R,S et R-S. ${ }^{2}$ S'y rajoutent la position de $\mathrm{E}$ par rapport à $\mathrm{R}$, ce qui donne, pour le français, deux sous-systèmes temporels morphologiquement motivés :
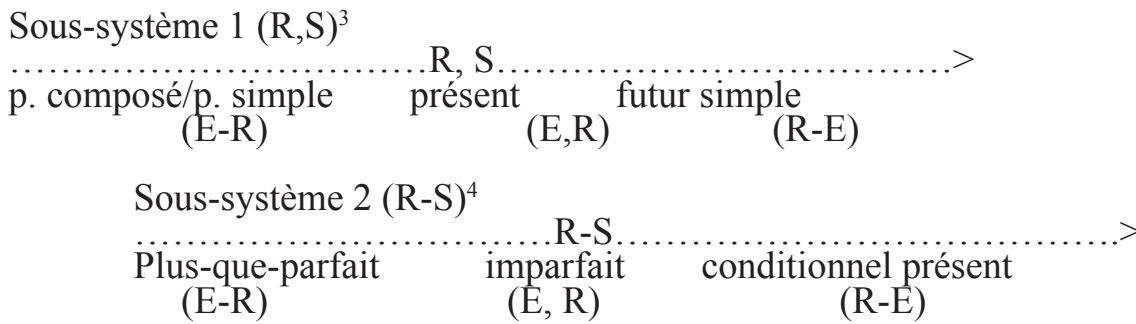

\footnotetext{
2 Pour les arguments contre S-R voir Vet (2008)

${ }^{3}$ Pour plus de lisibilité, sont absents du premier sous-système quelques temps verbaux, à savoir : le futur antérieur qui est la version résultative du futur simple (c'est-à-dire $S, R$ и R-E $E_{\text {RÉS }}$ ), le futur périphrastique, qui peut exprimer l'aspect prospectif (cf. Vet 2008) défini par les conditions : R,S et R,PRÉP (où $_{\mathrm{PRÉ}} \mathrm{E}_{\mathrm{E}}$ symbolise la phase préparatoire, c'est-à-dire la phase qui précède la réalisation de l'action et qui est en vigueur au moment de perspective temporelle) et le passé surcomposé (ex. Quand il a eu mangé, il est sorti.), lequel combine le trait d'antériorité et le trait résultatif dans le passé $\left(S, R\right.$ et $\left.E_{R E ́ S}-R\right)$. Toutes ces formes verbales font, donc, partie intégrante du sous-système 1 , étant donné que le procès qu'elles désignent est localisé par rapport au moment de perspective temporelle coïncidant avec le moment de la parole.

4 Pour plus de lisibilité, sont absentes du second sous-système quelques formes verbales dotées non seulement d'une valeur temporelle mais aussi d'une valeur aspectuelle (aspect résultatif ou prospectif), à savoir le conditionnel passé (R-S et R-E $\mathrm{RÉ}_{\mathrm{S}}$ ), le futur périphrastique du passé (ex. Il a dit qu'il allait le faire), qui a pour définition R-S et R, PRÉP $\mathrm{E}_{\mathrm{E}}$, et le plus-que-parfait composé (R-S et $\mathrm{E}_{\mathrm{RÉS}}-\mathrm{R}$ ), qu'on trouve en corrélation avec le plus-que-parfait (ex. Quand il avait eu mangé, il était sorti.).
} 
Le rapport de $\mathrm{R}$ à $\mathrm{S}$ est un élément de l'ensemble des traits sémantiques dont la conjonction engendre les temps verbaux du français. En effet, chaque temps verbal aurait deux traits temporels dont le premier encode la position de $\mathrm{R}$ par rapport à $\mathrm{S}$ et l'autre la position de $\mathrm{E}$ par rapport à $\mathrm{R}$. Le premier trait est un élément de l'ensemble \{PRÉS, PASSÉ\} où PRÉS correspond à la relation R,S et PASSÉ à la relation R-S. Le deuxième trait fait partie de l'ensemble \{ANT, POST, $\varnothing\}$ dont les éléments encodent la localisation temporelle, c'est-à-dire la position de E par rapport à R. ${ }^{5}$ Par ANT on entend la relation E-R, par POST la relation R-E, par $\varnothing$ la relation R,E par défaut.

Toujours est-il qu'en français la position de $\mathrm{R}$ reste fixe dans les différents emplois d'un même temps verbal.

Ce travail a pour but de montrer, d'un côté, qu'il y a des langues, comme le serbe, dans lesquelles la position de $\mathrm{R}$ n'est pas fixe et, de l'autre côté, que les différences d'emploi des temps verbaux en français et en serbe proviennent, dans une large mesure, du statut de $\mathrm{R}$ dans les deux langues. Nous nous focaliserons sur les temps verbaux du serbe pour lesquels il s'avère nécessaire de postuler la flexibilité de la perspective temporelle en fonction du contexte. La prise en compte d'un même ensemble de traits, partagés par les deux langues, nous permettra de prédire les équivalents français des temps serbes dont nous postulons la flexibilité de R.

\section{Un argument en faveur de la flexibilité de la perspective temporelle en serbe}

Considérons maintenant le système temporel du serbe. Il semble que certains temps verbaux de cette langue aient des interprétations dont on ne peut pas rendre compte si l'on suppose que le procès (E) est localisé toujours par rapport à une perspective temporelle $(\mathrm{R})$ dont la position est fixe sur l'axe du temps. Prenons l'exemple du parfait imperfectif (le parfait des verbes imperfectifs). Ce temps peut exprimer des actions en cours ou en vigueur à un moment du passé, ce qui sous-entend qu'au moins pour ce type d'emplois ce moment fonctionne comme R (R-S). Il est à noter que dans ce cas-là le parfait imperfectif (désormais $\mathrm{PI}$ ) a pour équivalent l'imparfait français (R-S et E,R).

4) Kad sam se vratio (R) ona je pravila kolače (E). (Quand je suis rentré (R) elle faisait des gâteaux (E).)

5) U tom trenutku (R) čitao sam knjigu (E). (A ce moment-là (R), je lisais un livre (E).)

\footnotetext{
${ }^{5}$ L'absence de trait marquée par le symbole $\varnothing$ est significative aussi.
} 
En effet, pour exprimer des actions en cours dans le passé les deux langues disposent chacune d'une forme qui exprime la simultanéité de $\mathrm{E}$ avec $\mathrm{R}$ (plus précisément $\mathrm{E}$ en cours ou en vigueur à $\mathrm{R}$ ), le moment de perspective $\mathrm{R}$ étant dans le passé (R-S). Ce sont bien le PI serbe et l'IMP français qui, dans ce cas de figure, assurent une vision imperfective du procès, ce qui veut dire que seule compte la phase de E qui est effective au moment R. Dit autrement, on se focalise sur la phase médiane du procès. Selon Vet, L'IMP français se définit par les conditions suivantes : R-S, pour la perspective temporelle et E,R (par défaut), pour la localisation temporelle. On dira donc que l'imparfait français localise, par défaut, la phase médiane du procès dans le passé. Au vu des exemples (4) et (5), le PI serbe semble fonctionner comme l'imparfait français, d'autant plus qu'on peut supposer que le rôle de l'aspect imperfectif - qui en serbe, comme dans les autres langues slaves est encodée déjà au niveau lexical et/ou dérivationnel - est de localiser la phase médiane de $\mathrm{E}$ dans le passé (c'est-à-dire comme étant en cours/en vigeur à $\mathrm{R}$ ).

Cependant, les choses ne sont pas aussi simples que cela. En effet, dans certains cas, lors de l'usage du PI, la perspective temporelle semble être non pas dans le passé, mais au moment de la parole $(\mathrm{S})$. Le procès (E) reste en lien avec $\mathrm{S}$, ce dont temoigne l'exemple suivant, dans lequel l'équivalent français du PI est le passé composé (PC) résultatif :

6) Pravila je (PI) kolace. Dođi da ih probaš. (Elle a fait des gâteaux. Viens les goûter)

7) Čitao sam (PI) tu knjigu. Mnogo mi se dopala. (J'ai lu ce livre. Je l'ai beaucoup aimé.) R,S et E-R

Notons au passage que le parfait imperfectif, dit le parfait d'expérience, (Stanojević et Đurić 2019) présuppose aussi que R est dans S :

8) Gledao sam taj film. (J'ai regardé ce film.), Bio sam u Parizu. (J'ai été à Paris.), Čitao sam Balzaka. (J'ai lu Balzac.), etc.

On supposera que la relation $\mathrm{R}, \mathrm{S}$ dans le cas du parfait imperfectif favorise la présentation globale du procès (focalisation sur le procès entier), alors que R-S (imposé par le contexte) en favoriserait la vision imperfective. ${ }^{6}$ Rappelons que dans les deux cas on utilise la forme imperfective du verbe lexical. En fonction de la position de $\mathrm{R}$ par rapport à $\mathrm{S}$, des formes lexicalement imperfectives comme praviti (faire) ou čitati (lire) peuvent désigner: a) des actions en cours dans le passé si la perspective dans le passé (c'est-à-dire R-S) est introduite par un loca-

\footnotetext{
${ }^{6}$ Pourtant, dans la section suivante nous allons voir que la présentation globale du procès n'est pas exclue même si la perspective est dans le passé (R-S).
} 
lisateur temporel (un adverbial de localisation temporelle ou une subordonnée temporelle) auquel cas le PI fonctionne comme imparfait français imposant au procès une vision imperfective (focalisation sur la phase médiane du procès) ou b) des actions complètement terminées dans le passé, si la perspective temporelle est dans $\mathrm{S}(\mathrm{R}, \mathrm{S})$. Dit autrement, le PI peut référer, suivant le contexte, soit à la phase médiane du procès, soit à la totalité de celui-ci.

Par ailleurs, on admettra que la combinaison 'auxiliaire + participe passé' peut introduire, selon le contexte: a) un E antérieur à R (voir exemple 9) auquel cas sera effective la lecture globale du PI, ou b) l'état résultant de $\mathrm{E}\left(\mathrm{E}_{\text {RÉS }}\right)$ valable à $\mathrm{S}$ (voir les exemples 6 et 7 ci-dessus) auquel cas sera en vigueur la lecture résultative du PI.

9) Jutros je dolazio poštar. (Le facteur est passé ce matin.) E-R et R,S

L'important à souligner est que l'aspect imperfectif ne bloque pas cette lecture globale du PI. On dira que, dans le cas du PI, R est par défaut au moment de la parole. Ce sont des facteurs contextuels qui peuvent faire déplacer $\mathrm{R}$ dans le passé en imposant une vision imperfective au procès $(\mathrm{E}, \mathrm{R})$ et notamment lors de l'usage d'adverbiaux de localisation temporelle ou de subordonnées temporelles (comme dans les exemples 4 et 5 ci-dessus).

\subsection{Le cas du parfait perfectif}

Le parfait perfectif (PP) du serbe a, lui aussi, un double fonctionnement. Il aurait par défaut la même lecture temporelle que le PI serbe (ou le PC français), ce qui veut dire qu'il introduirait les conditions suivantes: R,S (par défaut) et E-R (voir exemple 10). Cependant, dans certains cas le PP se comporte comme le plusque-parfait (PQP) français (comme dans l'exemple 11) avec lequel la perspective temporelle (R) est dans le passé. ${ }^{7}$ Donc, tout comme le PI, le PP est, lui aussi, ambivalent concernant sa sémantique temporelle. Cette ambivalence est fonction de la position de $\mathrm{R}$ en serbe qui semble flexible à la différence du français.

10) Juče je otišla. (Hier, elle est partie.) R,S et E-R

11) Rekao mi je da je otišla. (Il a dit qu'elle était partie.) R-S et E-R

Il est important de souligner que dans le cas du PP, le changement de la position de $\mathrm{R}$ se fait uniquement par le recours à un verbe d'attitude propositionnelle (comme dans l'exemple 11). Le rapport de $\mathrm{E}$ à $\mathrm{R}$ dans le passé reste alors le même que le rapport de $\mathrm{E}$ à $\mathrm{R}=\mathrm{S}$ (E-R). Le $\mathrm{PP}$ exprime toujours l'antériorité par rapport à la perspective temporelle où qu'elle se trouve (à $\mathrm{S}$ ou avant $\mathrm{S}$ ).

\footnotetext{
${ }^{7}$ En français, avec le $\mathrm{PC}$ la perspective est à $\mathrm{S}$, avec le $\mathrm{PQP}$ elle est dans le passé
} 
Dans les autres cas (usage de localisateurs temporels ou de subordonnées temporelles) $\mathrm{R}$ reste dans $\mathrm{S}$, le localisateur précisant tout juste le moment de $\mathrm{E}$ (comme dans les exemples 12 et 13).

12) U pet sati je krenula. (Elle est partie à cinq heures.) E-R et $R, S$

13) Kad sam se vratio, ona je krenula. (Quand je suis rentré, elle est partie.) E-R et $\mathrm{R}, \mathrm{S}$

Par ailleurs, dans le cas du PI, la perspective change de position (R-S) soit sous l'effet d'un verbe d'attitude propositionnelle auquel cas le PI exprime le même rapport avec $\mathrm{R}$ que si R était au moment de la parole (l'antériorité) comme dans l'exemple (14), soit sous l'effet d'un localisateur temporel (adverbial ou subordonnée temporelle) comme dans les exemples (4) et (5) que nous reproduisons sous (15) et (16), auquel cas le PI exprime la simultanéité avec R.

14) Rekla mi je da je pravila kolače. (Elle m'a dit qu'elle avait fait des gâteaux.)

15) Kad sam se vratio (R) ona je pravila kolače (E). (Quand je suis rentré (R) elle faisait des gâteaux (E).)

16) U tom trenutku (R) čitao sam knjigu (E). (A ce moment-là (R), je lisais un livre (E).)

Donc, le PI tout comme le PP exprime l'antériorité par rapport à R si R est dans $\mathrm{S}$ ou si $\mathrm{R}$ dans le passé est introduit par un verbe d'attitude propositionnelle. Dans les autres cas, c'est-à-dire si R dans le passé n'est pas introduit par un verbe d'attitude propositionnelle, le PI exprime la simultanéité de E avec R (E en cours ou en vigueur à $\mathrm{R}$ ).

En plus de la lecture événementielle (comme en 10), le PP peut avoir aussi la lecture résultative (voir 17) auquel cas le locuteur se focalise non plus sur $\mathrm{E}$ mais sur $\mathrm{E}_{\text {RéS }}$ valable à R,S (cf. Tanasić $2005: 392$ ).

17) Gde je Marija? - Izašla je. (Où est Marie ? - Elle est sortie.) : $\mathrm{E}_{\mathrm{RÉS}}, \mathrm{R}$ et R,S

Toujours est-il qu'en serbe il faut supposer que R est flexible au moins pour le parfait indépendamment de l'aspect verbal. On verra dans la section suivante qu'il faut supposer aussi la flexibilité de $\mathrm{R}$ dans le cas d'autres temps verbaux formés à partir de la morphologie du présent, comme le présent imperfectif ou le futur I. Avant de le faire, nous présenterons le système temporel du serbe en prêtant particulièrement attention au rôle qu'y joue la perspective temporelle (R). 


\section{Le système temporel du serbe}

Nous supposons pour le serbe aussi deux sous-systèmes, dont seul le premier, basé sur la morphologie du présent est complet (le présent, le parfait et le futur I), alors que le deuxième est incomplet parce qu'il comprend seulement le $\mathrm{PQP}$, formé à partir du parfait de l'auxiliaire biti et du participe passé. Nous supposerons que la fonction du parfait de l'auxiliaire est de déplacer $\mathrm{R}$ dans le passé, auquel E est antérieur.
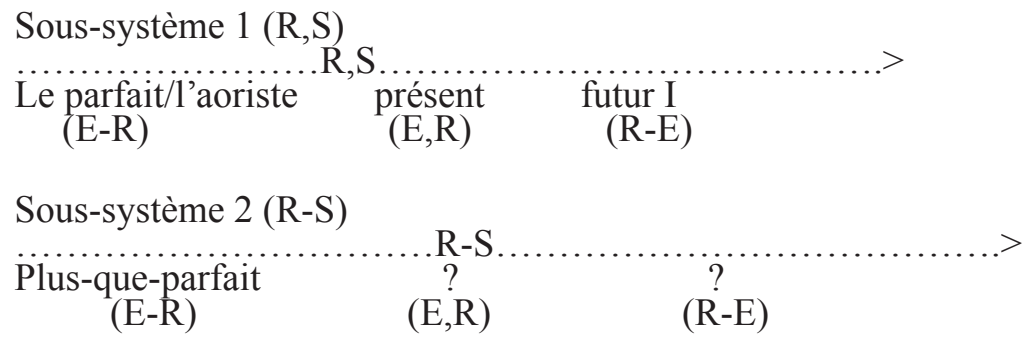

Le deuxième sous-système temporel (avec la perspective temporelle dans le passé) en serbe présente des lacunes morphosyntaxiques, ce qui veux dire qu'il n'existe pas de forme verbale spécifique pour exprimer les relations E,R et R-E. Dit autrement, le système n'engendre pas de nouveaux temps verbaux pour signaler les relations E,R et R-E, si R est dans le passé. Il suffit de considérer les exemples suivants pour se rendre compte que les lacunes morphosyntaxiques du $2^{\text {ème }}$ sous-système sont comblées par les TV du premier sous-système du serbe :

18) Rekao je (R) da je bolestan (E). (Il a dit qu'il était malade.) R-S et R,E

19) Rekao je (R) da će doći (E). (Il a dit qu'il viendrait.) R-S et R-E

Dans les travaux de linguistes serbes on parle ici d'emplois relatifs du présent et du futur I (cf. Tanasić $2005: 365-370$ et 437-438). En français, on utilisera dans ce cas les temps du $2^{\text {ème }}$ sous-système (voir section 1). En serbe, par contre, l'inexistence de formes spécifiques pour exprimer les relations E,R et R-E (avec $\mathrm{R}$ dans le passé) explique que soient empruntées ici, pour combler ces lacunes, les temps du premier sous-système exprimant les mêmes relations entre E et R. C'est pourquoi nous postulons que dans le cas des temps du premier sous-système du serbe la relation R,S est une option par défaut illustrant les emplois qu'on pourra qualifier de déictiques ${ }^{8}$ des temps verbaux.

8 Ou indicatifs selon la terminologie des linguistes serbes (cf. Belić 1958, Stevanović 1967, Sladojević 1966, Tanasić 2005). 
Comme on l'a vu dans la section précédente le parfait serbe peut aussi avoir des emplois relatifs en dépit de l'existence du PQP qui marque l'antériorité par rapport à $\mathrm{R}$ dans le passé. Les exemples (11) et (20) illustrent ce type d'usage du PP en présence d'un verbe d'attitude propositionnelle. L'explication pourrait être la suivante. Comme le $\mathrm{R}$ dans le passé, introduit par un verbe d'attitude propositionnelle, fonctionne pour la localisation de E comme le moment de la parole, le PQP serbe n'est pas approprié pour signaler ce type d'antériorité. Toujours est-il que l'équivalent français d'un tel usage du PP est le PQP qui marque l'antériorité par rapport à $\mathrm{R}$ dans le passé de quelque manière que $\mathrm{R}$ soit introduit:

20) Rekao je da je to uradio. (Il a dit qu'il avait fait cela) R-S et E-R

On a vu également dans la section précédente que le PI peut avoir le même type d'emploi (antériorité par rapport à un $\mathrm{R}$ dans le passé) lorsque $\mathrm{R}$ est introduit par un verbe d'attitude propositionnelle:

21) Rekao mi je da je već čitao tu knjigu. (Il m’a dit qu’il avait déjà lu ce livre) : $\mathrm{R}-\mathrm{S}$ et $\mathrm{E}-\mathrm{R}$

L'action de lire ce livre est presentée à la fois comme terminée dans le passé et comme antérieure par rapport au procès fonctionant comme $\mathrm{R}$ (il m'a dit). ${ }^{9}$

De ce qui précède découle l'observation suivante : si la perspective temporelle dans le passé est introduite par un verbe d'attitude propositionnelle, alors, pour localiser un $\mathrm{E}$, on doit faire comme si R était à $\mathrm{S}$. Autrement dit, si un $\mathrm{R}$ dans le passé est introduit par un verbe d'attitude propositionnelle, il fonctionnera pour la localisation temporelle comme $\mathrm{S}$, ce qui implique qu'on utilisera alors les temps du premier sous-système pour marquer les relations de simultanéité, de postériorité ou d'antériorité. Le présent désignera alors la simultanéité de $\mathrm{E}$ avec $\mathrm{R}$, le parfait l'antériorité et le futur I la postériorité de $\mathrm{E}$ par rapport à $\mathrm{R}$. Si la perspective temporelle $(\mathrm{R})$ dans le passé est introduite d'une autre façon, la simultanéité de $\mathrm{E}$ avec $\mathrm{R}$ ne sera exprimée que par le parfait imperfectif et l'antériorité par le $\mathrm{PQP}^{10}$. Le futur I n'exprime la postériorité par rapport à un $\mathrm{R}$ dans le passé que si $\mathrm{R}$ est introduit par un verbe d'attitude propositionnelle.

\footnotetext{
${ }_{9}$ Rappelons que si R dans le passé n'est pas introduit par un verbe d'attitude propositionnelle ce n'est pas l'antériorité mais la simultanéité de $\mathrm{E}$ et de R qui est en vigueur, auquel cas l'équivalent français du PI sera l'imparfait comme dans les exemples (4) et (5) ou dans les exemples suivants:

i) Čitao je tu knjigu (E) kad sam ušao (R). (Il lisait ce livre (E) quand je suis entré (R).)

ii) Marko uđe (R) u sobu. Marija je spavala (E). (Marco entra (R) dans la chambre. Marie dormait (E).)

${ }^{10} \mathrm{Kad}$ me je nazvala, ja sam već bio otputovao iz Pariza. U tom trenutku ja sam već bio otputovao iz Pariza. (Quand elle m'a appelé j'étais déjà parti de Paris. A ce moment-là je j'étais déjà parti de Paris.)
} 


\section{Sémantique des temps du premier sous-système en serbe}

Nous allons proposer une définition pour chaque temps verbal du premier sous-système temporel en serbe en adoptant l'hypothèse sur la flexibilité du moment de perspective en serbe. En admettant qu'avec les temps du premier soussystème la perspective temporelle puisse changer de place nous indiquerons pour chacun des temps verbaux concernés les deux possibilités (R,S et R-S) et préciser pour chacune la position de $\mathrm{E}$ par rapport à $\mathrm{R}$ ainsi que des facteurs imposant cette dernière et notamment dans les cas où $\mathrm{R}$ précède $\mathrm{S}$.

En suivant Vet (2008) nous supposerons que la relation R,S correspond au trait (ou opérateur) temporel PRÉS, alors que la relation R-S correspond au trait PASSÉ. PRÉS et PASSÉ représentent donc deux manières d'encoder la position de la perspective temporelle dans la définition sémantique des temps verbaux. Aux relations entre $\mathrm{E}$ et $\mathrm{R}$ (relations encodant la localisation temporelle) correspondent les traits ANT (E-R) et POST (R-E). L'absence de trait de localisation temporelle sera noté $\varnothing$ dont l'interprétation par défaut est la simultanéité de $\mathrm{E}$ et de $\mathrm{R}(\mathrm{E}, \mathrm{R})$. Pour rendre compte de la lecture résultative nous introduirons, comme Vet, le trait RÉS qui signifie que l'accent communicatif est mis sur la phase post-finale du procès (qu'on notera $\mathrm{E}_{\mathrm{RÉS}}$ ).

\section{Présent :}

$<$ PRÉS, $\varnothing>$ (option par défaut, E simultané ${ }^{11}$ avec R qui est à $\left.S\right)^{12}$, ou

$<$ PASSÉ, $\varnothing>$ si R est introduit par un verbe d'attitude propositionnelle ${ }^{1314,}$

Parfait imperfectif :

$<$ PRÉS, ANT > (c'est-à-dire, E antérieur à R qui est à S), ou

$<$ PRÉS, RÉS > (c'est-à-dire, $\mathrm{E}_{\text {RÉS }}$ simultané avec R qui est à $\mathrm{S}$ ), ou

$<$ PASSÉ, ANT $>$, si R est introduit par un verbe d'attitude propositionnelle. Sinon, $<$ PASSÉ, $\varnothing>$.

\footnotetext{
${ }^{11} \varnothing$ est une option par défaut qui peut être contextuellement changée : On dolazi. (= On će doći) / On je rekao da dolazi (=on je rekao da će doći): R-E; E-R dans le cas du présent à référence future.

${ }^{12}$ Cela se lit: lors de l'emploi du présent si R est au moment de la parole, alors E,R (défaut)

13 C'est-à-dire, E simultané avec R dans le passé, si R est introduit par un verbe d'attitude propositionnelle.

${ }^{14}$ On pourrait rajouter aussi le discours indirect libre en tant que facteur déclenchant la simultanéité de $\mathrm{E}$ et $\mathrm{R}$, mais pour simplifier la présentation nous avons omis ce facteur dans la définition des temps verbaux du serbe.
} 
Parfait perfectif :

$<$ PRÉS, ANT $>$, ou

$<$ PRÉS, RÉS $>$, ou

$<$ PASSÉ, ANT $>$, si R est introduit par un verbe d'attitude propositionnelle

Futur I :

$<$ PRÉS, POST $>$ ou

$<$ PASSÉ, POST $>$

Les définitions proposées des temps du premier sous-système en serbe nous permettent de prédire leurs équivalents français en nous fondant sur un même ensemble de traits sémantiques concernant la position de $\mathrm{R}$ par rapport à $\mathrm{S}$ ainsi que celle de $\mathrm{E}$ par rapport à $\mathrm{R}$. Comme on l'a déjà vu à la section 1 de cet article, ces traits sémantiques, tels que proposés par Co Vet (Vet 2008), sont les suivants : PRÉS, PASSÉ (pour la perspective temporelle), ANT, POST (pour la localisation temporelle) auxquels Vet ajoute deux traits aspectuels pertinents pour le français, à savoir RÉS et PROSP. Cependant, à la différence de Vet et pour simplifier la présentation, nous postulerons que RÉS, tout en étant un trait aspectuel signifiant la focalisation sur la phase post-finale du procès, peut servir aussi à localiser l'état résultant du procès $\left(\mathrm{E}_{\mathrm{RÉS}}\right)$ par rapport à $\mathrm{R}$, c'est-à-dire à établir une relation temporelle entre l'état résultant et $\mathrm{R}$. Nous ferons l'hypothèse qu'en l'absence d'un des traits $\mathrm{ANT}^{15}$ ou POST pour la localisation temporelle, cette relation sera par défaut celle de simultanéité entre $\mathrm{E}_{\mathrm{RÉS}}$ et $\mathrm{R}\left(\mathrm{E}_{\mathrm{RÉS}}, \mathrm{R}\right)^{16}$, signifiant que l'état résultant de $\mathrm{E}$ est effectif à $\mathrm{R}$. Autrement dit, si résultativité il y a, l'antériorité ou la postériorité étant exclue ${ }^{17}$, le trait RÉS signalera par défaut que l'état résultant du procès est valable à $R$. Nous pouvons maintenant prédire les équivalents français des temps du premier sous-système serbe (voir Tableau 1 ci-dessous) en nous basant sur les instructions temporelles encodées en termes de traits sémantiques pertinents pour l'interprétation dans les deux langues.

\footnotetext{
${ }_{15}$ Dans le système temporel du français les traits ANT et RÉS ne coexistent comme composantes sémantiques que dans le cas du passé antérieur et des temps surcomposés (Vet $2010: 21$ ), alors que POST et RÉS ne font partie que du sémantisme du futur antérieur. Dans le cas du passé composé et du plus-que-parfait, ANT et RÉS sont en distribution complémentaire, ce qui signifie que la lecture événementielle (imposée par le trait ANT) exclut la lecture résultative (imposée par le trait RÉS) et inversement (la lecture résultative exclut la lecture événementielle). Il en découle que la présence du seul trait RÉS impose (par défaut) la simultanéité de l'état résultant $\mathrm{E}_{\mathrm{RÉS}}$ avec le moment de perspective temporelle ( $\left.\mathrm{E}_{\mathrm{RÉ} S}, \mathrm{R}\right)$.

16 Tout comme elle l'est aussi, dans le système de Vet, entre E et R, ce que nous nous notons, en suivant Vet par l'opérateur zéro $(\varnothing)$.

17 Par exemple, si le contexte implique qu'on s'interroge sur un état de choses valable au moment de la parole, le passé composé, utilisé pour nous informer sur cet état de choses, aura la lecture résultative et non pas événementielle. C'est, par exemple, le cas du passé composé dans la phrase Il est parti en réponse à la question Où est-t-il ?
} 
Tableau 1 : Les temps du premier sous-système en serbe et leurs équivalents français

\begin{tabular}{|c|c|c|c|}
\hline & $\begin{array}{c}\mathrm{PT}^{18} \text { (rapport de } \\
\mathrm{R} \text { à S) }\end{array}$ & 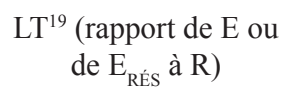 & Équivalents français \\
\hline PRÉSENT & $\begin{array}{c}\text { PRÉS (R,S) } \\
\text { PASSÉ (R-S), R } \\
\text { introduit par un } \\
\text { verbe d'attititude } \\
\text { propositionnelle }\end{array}$ & $\begin{array}{c}\varnothing(=\mathrm{E}, \mathrm{R}) \\
\varnothing \ldots \ldots \ldots \ldots \ldots \\
\varnothing(=\mathrm{E}, \mathrm{R})\end{array}$ & $\begin{array}{c}\text { Présent (PT: PRÉS ; LT: } \\
\varnothing) \\
\text {.................................... } \\
\text { LT: Ø) }\end{array}$ \\
\hline $\begin{array}{c}\text { PARFAIT } \\
\text { IMPERFECTIF }\end{array}$ & PASSÉ (R-S) & $\begin{array}{c}\text { ANT (=E-R) } \\
\left.\text { RÉS (=E } E_{\text {RÉS }}, \mathrm{R}\right) \\
\text {....................... } \\
\text { ANT (=E-R), si R } \\
\text { est introduit par } \\
\text { un verbe d'attitude } \\
\text { propositionnelle) } \\
\varnothing \text { (=E,R), si R est } \\
\text { introduit autrement } \\
\text { que par un verbe } \\
\text { d'attitude propos. }\end{array}$ & $\begin{array}{c}\text { P. composé (PT: PRÉS; LT: } \\
\text { ANT) } \\
\text { P. composé (PT: PRÉS; LT: } \\
\left.\left.\text { RÉS (= } E_{\text {RÉS }}, \mathrm{R}\right)\right) \\
\text { Plus-que parfait (PT: } \\
\text { PASSÉ; LT: ANT) } \\
\text { Imparfait (PT: PASSÉ; LT: } \\
\varnothing)\end{array}$ \\
\hline $\begin{array}{c}\text { PARFAIT } \\
\text { PERFECTIF }\end{array}$ & $\begin{array}{c}\text { PRÉS (R,S) } \\
\text { PASSÉ (R-S), R } \\
\text { est introduit par } \\
\text { un verbe d'attit. } \\
\text { propos. }\end{array}$ & $\begin{array}{c}\text { ANT (=E-R) } \\
\text { RÉS (= } \text { EÉs }, R) \\
\text { ANT (=E-R) }\end{array}$ & $\begin{array}{c}\text { P. composé (PT: PRÉS; LT: } \\
\text { ANT) } \\
\text { P. composé (PT: PRÉS; LT: } \\
\text { RÉS (E } \text { EÉs }^{\text {R R) }} \\
\text { Plus-que parfait (PT: } \\
\text { PASSÉ; LT: ANT) }\end{array}$ \\
\hline FUTUR I & $\begin{array}{c}\text { PRÉS (R,S) } \\
\text {..................... } \\
\text { PASSÉ (R-S), } \\
\text { R est introduit par } \\
\text { un verbe d'attit. } \\
\text { propos. }\end{array}$ & $\begin{array}{l}\text { POST (=R-E) } \\
\text { POST (=R-E) }\end{array}$ & $\begin{array}{c}\text { Futur simple/fut. } \\
\text { périphrastique en emploi } \\
\text { temporel (PT: PRÉS; LT: } \\
\text { POST) }\end{array}$ \\
\hline
\end{tabular}

\footnotetext{
${ }_{18}$ Perspective temporelle (rapport de R à S).

${ }^{19}$ Localisation temporelle (rapport de E à R).
} 


\section{En guise de conclusion}

Le fait qu'un même temps du serbe puisse avoir au moins deux équivalents français est dû, au niveau sémantique, à la flexibilité de la perspective temporelle et notamment dans le cas des temps du premier sous-système temporel du serbe. Par ailleurs, en français, la perspective temporelle est fixe : elle est située, pour chaque forme verbale fléchie, soit au moment de la parole, soit à un moment du passé. Intuitivement, cela veut dire qu'à la différence du français, les temps basés sur la morphologie du présent, qui constituent un premier sous-système des temps verbaux en serbe, localisent le procès (E) par rapport au moment de perspective (R) qui, en fonction du contexte, peut changer de position par rapport à $\mathrm{S}$. Le déclencheur typique d'un tel déplacement de $\mathrm{R}$ est la présence d'un verbe d'attitude propositionnelle au passé, ce qui fait que pour localiser le procès de la subordonnée dans le temps on utilisera, en fonction du rapport temporel entre $\mathrm{E}$ et $\mathrm{R}$, un des temps verbaux du premier sous-système temporel.

Cette flexibilité de $\mathrm{R}$ en serbe est sans doute due aux lacunes morphosyntaxiques du deuxième sous-système temporel du serbe et notamment en ce qui concerne l'expression de la simultanéité et de la postériorité du procès dans le passé. Il est intéressant de constater que seulement dans le cas du parfait imperfectif la présence d'un localisateur temporel, à savoir d'une subordonnée ou d'un adverbial temporel, peut induire un changement de la position de $\mathrm{R}$ avec pour effet de sens un changement automatique de la localisation temporelle, qui n'est plus celle d'antériorité, mais de simultanéité de E avec R.

Faute de place, nous n'avons pu examiner des facteurs d'ordre discursif qui, eux aussi, peuvent bien jouer un rôle dans le déplacement de $\mathrm{R}$ en serbe, et notamment les relations temporelles entre deux ou plusieurs phrases constitutives d'un discours cohérent. Le rôle de l'aspect verbal lors du changement de la position de $\mathrm{R}$ reste également à explorer d'autant plus qu'on a pu constater des différences entre le parfait perfectif et le parfait imperfectif quant aux possibilités de localisation temporelle (rapport de E à R, si R est dans le passé).

\section{Références bibliographiques}

Belić 1958 : A. Belić, O jezičkoj prirodi i jezičkom razvitku, Beograd : Nolit.

Sladojević 1966: P. Sladojević, O osnovnim vremenskim kategorijama upotrebe glagolskih oblika u srpsko-hrvatskom jeziku, Beograd: Naučna knjiga.

Stanojević et Ašić 2010 : V. Stanojević, T. Ašić, « L'aspect imperfectif en français et en serbe », Interpréter les temps verbaux, in Nelly Flaux, Dejan Stošić, Co Vet (dir.), Frankfurt : Peter Lang, 107-129. 
Stanojević et Đurić 2019 : V. Stanojević, Lj. Đurić, « Aspekat kao fazna kategorija i rezultativnost u francuskom i srpskom jeziku », Srpski jezik 24 (à paraître).

Stevanović 1967 : M. Stevanović, Funkcije i značenja glagolskih vremena, Beograd : Naučno delo.

Tanasić 2005 : S. Tanasić, 'Sintaksa glagola’, In: P. Piper, I. Antonić, V. Ružić, S. Tanasić, Lj. Popović, B. Tošović, Sintaksa savremenog srpskog jezika: prosta rečenica, Beograd: Institut za srpski jezik SANU, Beogradska knjiga - Novi Sad : Matica srpska, 345-475.

Vet 1980 : Co Vet, Temps, aspects et adverbes de temps en français contemporain. Geneva : Droz.

Vet 2008 : Co Vet, « Six traits sémantiques suffisent à décrire tous les temps du français », in M. Birkelund, M.-B. Mosegaard Hansen et C. Norén (dir.), L'énonciation dans tous ses états : Mélanges offerts à Henning Nølke à l'occasion de ses soixante ans, Berne : Peter Lang.

\section{Веран Станојевић}

\section{Флексибилност временске перспективе и темпорални системи у француском и српском језику}

У раду испитујемо улогу временске перспективе у интерпретацији глаголских времена у српском у поређењу са француским језиком. Ослањајући се на Ветов приступ по којем, у француском, временска перспектива може бити или у моменту говора или у прошлости, али не мења своју позицију без обзира на различите временске употребе глаголских облика, констатовали смо да је у српском неопходно претпоставити флексибилност временске перспективе како би се објасниле различите употребе финитних глаголских облика првог темпоралног подсистема, тј. презента, перфекта и футура. Тиме се могу објаснити релативне употребе поментих глаголских времена у српском, али и предвидети њихови француски еквиваленти и то без обзира на позицију временске перспективе према моменту говора коју они, зависно контекста, могу индуковати.

Кључне речи: глаголска времена, глаголски вид, временска перспектива, српски језик, француски језик. 